Annales Geophysicae (2003) 21:787-796 (C) European Geosciences Union 2003

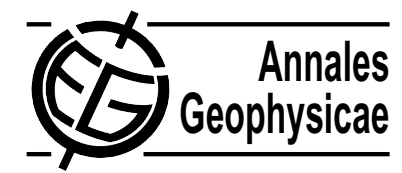

\title{
Periodicities in energy dissipation rates in the auroral mesosphere/lower thermosphere
}

\author{
C. M. Hall ${ }^{1}$, S. Nozawa ${ }^{2}$, C. E. Meek ${ }^{3}$, A.H. Manson ${ }^{3}$, and Yi Luo ${ }^{3}$ \\ ${ }^{1}$ University of Tromsø, Norway \\ ${ }^{2}$ Nagoya University, Japan \\ ${ }^{3}$ University of Saskatchewan, Canada
}

Received: 4 April 2002 - Revised: 28 August 2002 - Accepted: 26 September 2002

\begin{abstract}
It is possible for medium-frequency (MF) radar systems to estimate kinetic energy dissipation rates by measuring signal fading times. Here, we present approximately 5 years of such results from Troms $\emptyset\left(69^{\circ} \mathrm{N}, 19^{\circ} \mathrm{E}\right)$ and in particular, investigate the periodicities present at different altitudes in the regime 80 to $100 \mathrm{~km}$. We detect the known annual variation in the mesosphere and the semiannual variation on the lower thermosphere. In addition, other features are observed including terannual and $\sim 27$-day components in the lower thermosphere.
\end{abstract}

Key words. Meteorology and atmospheric dynamics (climatology; middle atmosphere dynamics; turbulence)

\section{Introduction: the data source}

The Universities' of Troms $\varnothing$, Saskatchewan and Nagoya joint MF radar system, operating at $2.78 \mathrm{MHz}$ at Ramfjordmoen $\left(69^{\circ} \mathrm{N}, 19^{\circ} \mathrm{E}\right)$ near Troms $\emptyset$ in northern Norway has been described recently by Hall (2001a). This wellestablished installation currently yields winds derived from the Full Correlation Analysis (FCA) (Meek, 1980; Briggs, 1984) of signals from each of 3 spaced receivers. In addition, the FCA determines signal fading times. It is then possible to estimate an upper limit, $\varepsilon^{\prime}$, to the turbulent energy dissipation, $\varepsilon$, from this echo fading time, by assuming either a length scale or a time scale corresponding to the largest scale of turbulence. It is common to adopt the latter, obtaining it from the reciprocal of the Brunt-Väisälä frequency, in turn obtained from a model or from prior observations. Whether or not this scale represents the largest scale of eddy or whether gravity waves are also present is impossible to know. Therefore, values derived by this method may include contributions from any gravity waves with periods the same as the Brunt-Väisälä period and, therefore, are usually thought of as upper limits for the true turbulent energy dissipation rate $\varepsilon$.

Correspondence to: C. M. Hall (chris.hall@phys.uit.no)
While the derivation of $\varepsilon^{\prime}$ and estimation of $\varepsilon$ are discussed in detail by Hocking (1999) and Roper (2000), we shall summarize the method here. The signal fading times, $\tau_{c}$ are determined by the FCA mentioned earlier. Subsequently, velocity fluctuations, $v^{\prime}$, appropriate to an observer moving with the background wind, are computed at a height resolution of $3 \mathrm{~km}$ via

$v^{\prime}=\frac{\lambda \sqrt{\ln 2}}{4 \pi \tau_{c}}$,

where $\lambda$ is the radar wavelength. In an ideal situation, the radar beam width would be infinitely narrow and exactly vertical. This not the case, however, and in order to allow for this we consider the measured $v^{\prime}$ to be the total component of the fluctuation. We have to assume that the fading time determined by the FCA is indeed the characteristic decay time of neutral atmosphere structures. Furthermore, although a structure seen by the radar is dissipating with a time constant $\tau_{c}$, we cannot assume a priori that it is exclusively the vertical (or horizontal) dynamics that are responsible. The energy dissipation rate may be arrived at by dividing the kinetic energy of the turbulence, related to $v^{\prime 2}$, by a time scale (Blamont, 1963). In previous studies, (e.g. Manson et al., 1981a) the Brunt-Väisälä period has been identified as a suitable time scale; the fluctuation is the vertical component, $U_{z}$, and the expression $\varepsilon=0.4 U_{z}^{2} \omega_{B}$ has been used $\left(\omega_{B}\right.$ being the Brunt-Väisälä frequency). Here, however, we have assumed a total velocity fluctuation and thus use

$\varepsilon^{\prime}=0.8 v^{\prime 2} / T_{B}$,

where $T_{B}$ is the Brunt-Väisälä period in seconds. Note that we recognise that the spatial scale inherent in the experiment cannot preclude buoyancy-scale fluctuations, because the outer scale of turbulence, $L_{B}$, can be expected to be as little as $200 \mathrm{~m}$ in the mesosphere. Again, this is why we choose to introduce $\varepsilon^{\prime}$ to distinguish our measurement from $\varepsilon$. Furthermore, Eq. (2) implicitly assumes there is no motion of the observer relative to the background wind; we have not attempted to compensate for departures from this. To use 


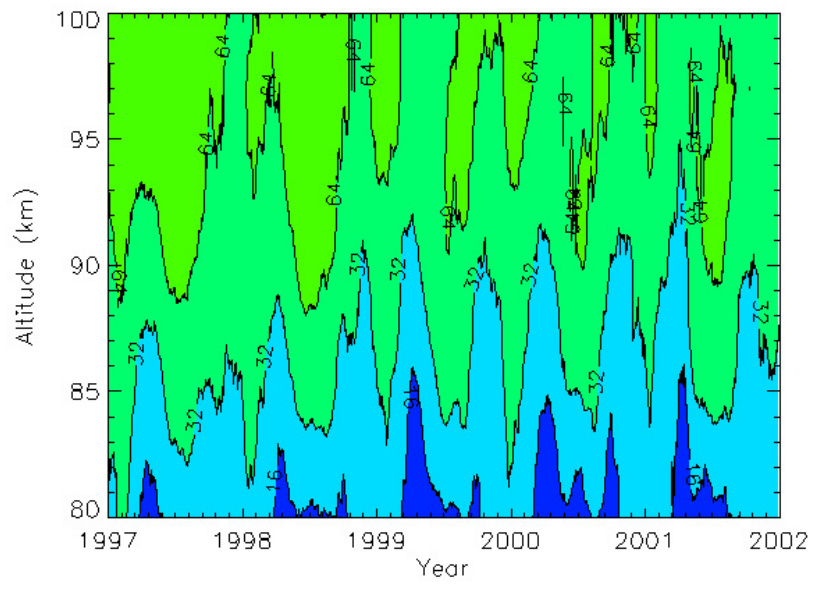

Fig. 1. Energy dissipation rate at the buoyancy scale, $\varepsilon^{\prime}$ (see text for details), as a function of time and height. The data shown here are the basis data set for the subsequent spectral analysis. Note that the heights, as for all figures, are virtual heights (see text).

a formulation for $\varepsilon$ which assumes a radar completely free from beam broadening here would be clearly inappropriate, and we attempt to address the warnings of Hocking (1983) by accepting that $v^{\prime}$ is an estimate of total velocity perturbations within the scattering volume, choosing a time scale accordingly. It should be pointed out, however, that a disadvantage of incorporating the Brunt-Väisälä period in the time scale is that we must use a model with implicit seasonal variation. The Brunt-Väisälä period is determined from the Mass Spectrometer and Incoherent Scatter (Extended) (MSIE90) model (Hedin, 1991).

Although there has been some dispute in the literature regarding the absolute values of the turbulent energy dissipation rate obtained by radar and in situ methods, the periodicities can be argued to be very similar. This is because, while the time scale implicit in the definition of $\varepsilon^{\prime}$ is indeed the period of the smallest gravity waves, it is, at the same time, the scale of the largest turbulent eddies. Even if turbulence is not present, energy is transferred from this scale into the viscous subrange (i.e. the inertial subrange vanishes). The exceptions to this scenario are when gravity waves are Doppler-shifted by the background wind in which they are embedded. Even so, their energy almost invariably cascades to smaller scales, as has been indicated by numerous in situ measurements. Lübken (1997) presents examples of spectra of neutral fluctuations and also provides references for virtually every in situ measurement of such spectra prior to 1994 from a location nearby Troms $\emptyset$. Further examples of the normality of the cascade to smaller scales are given by Blix et al. (1990).

Hall et al. (1998) resurrected the original study of the $\varepsilon^{\prime}$ parameter at Troms $\emptyset$ by Schlegel et al. (1980) and even suggested a spectral domain alternative method, provided sampling could span the Brunt-Väisälä frequency (Hall et al., 2000). In this study, we regard the latter method as experimental and restrict ourselves to the more established FCA method, despite the fact that it can be argued that the newer method may yield better estimates of $\varepsilon$ rather than $\varepsilon^{\prime}$. A recent paper by Holdsworth et al. (2001) addresses turbulent velocity estimation (the precursor to $\varepsilon^{\prime}$ calculation) and thoroughly explains the assumptions. As we have identified above, we are really estimating the kinetic energy fluctuation of the neutral atmosphere at the Brunt-Väisälä period and this fluctuation may be due to turbulence, gravity waves or both; rather than talk of turbulent energy dissipation we shall refer to just energy dissipation, but still retain the assumption of an energy cascade to smaller scales.

We have assembled data from December 1996 until the end of 2001. These 5 years of almost uninterrupted measurements contain both 5-min and 2-min records (i.e. the number of samples at each height assembled before the FCA is performed: 576 for 5 -min records and 240 for 2-min records); below around $70 \mathrm{~km}$ the 2-min integrated data exhibit more variability (we deign to use the term "noise"). However, after 1-day averaging, we are unable to see any systematic change in the overall energy dissipation rates at the times at which the integration times were changed. Meek and Manson (2001) discuss the influence of integration time on FCA in detail. Furthermore, the noise reduction algorithm of Meek (1990) has been applied to the signal fading times prior to calculation of $\varepsilon^{\prime}$. Figure 1 shows the values of $\varepsilon^{\prime}$ that we will use, as a function of time and height. The reliability of these results is, to some degree, a function of height: at the lowest heights echoes are weak due to a lack of ionisation, whereas above $90-100 \mathrm{~km}$, other problems arise, as we shall discuss. Heights are virtual (see Hall, 1998) and the spacing between them is $3 \mathrm{~km}$. The concept of "virtual height" is well-known to those involved with atmospheric sounders, such as MF radars and ionosondes and we summarise it here. The group velocity of a radio wave in travelling through the ionosphere depends both on the electron density and the radio wave frequency; therefore, as a wave of given frequency enters the ionosphere from below, it travels progressively slower as the electron density increases. Since we do not normally know the electron density profile a priori, an echo height calculation assumes the wave to be travelling at the speed of light. At high frequencies, such as VHF and $\mathrm{UHF}$, this is a reasonable assumption, however, at MF it is not. The heights at which the echoes are seen are, therefore, "virtual" and, depending on the degree of ionisation between the radar and the echo height, may be an overestimation of several kilometres. More detailed assessments of the consequences of group delay for the kinds of observations presented here have been given by Hall (1998) and Namboothiri et al. (1993). Indeed, when the degree of ionisation is sufficiently high, the radio wave is totally reflected. Group delay of the radar wave is only expected to be significant above $90 \mathrm{~km}$ and may be as much as a scale height at $120 \mathrm{~km}$ in individual soundings, depending on the electron density, and this is one of the reasons for restricting our study to altitudes below $100 \mathrm{~km}$. However, of the total number of individual soundings in this data set, only a small proportion is expected to be influenced by sufficiently high electron den- 
sities to cause large group delays. Statistically, therefore, the features seen in all the figures in this paper are assumed to be approximately representative of the heights shown on the ordinate axes. Furthermore, since many features are spread in height, i.e. the heights are not exact, is not an issue. The absolute values of the energy dissipation rate obtained from the FCA by the method used here (Hocking, 1999) have been discussed by Hall et al. (1999), in which they are compared with results from both in situ and other radar methods. We shall not concern ourselves with the absolute values here, therefore, and rather search for periodicities. Moreover, although $\varepsilon^{\prime}$ has been derived for the entire height coverage of the radar, the values obtained at heights above the turbopause must be assumed to be incorrect, or at least consequences of total reflection of the radar wave. Generally, the turbopause occurs around $120 \mathrm{~km}$ (varying with season and geomagnetic activity), so features we observe above this height must be thought of as pertaining to the "lower thermosphere" and not to any well-defined altitude. A further problem to be considered when interpreting signal fading times is that the structures responsible for radar backscatter may be dissipated by mechanisms other than neutral dynamics at altitudes at which ion drag becomes significant.

Finally, a recent study by Tsutsumi et al. (private communication, 2001 CEDAR/SCOSTEP Workshop) has shown that meteor echoes are likely to interfere with the ionisation structures at heights above $90 \mathrm{~km}$. The feature of such an echo most likely to affect the FCA is its quick rise time. This should raise the $\varepsilon^{\prime}$ value. However, given that enhanced signals only last a few seconds at MF, and that the receivers used have only a $20 \mathrm{~dB}$ dynamic range at any given height in a record, and that the receiver gains are continually changed between records to maintain the average signal in the midrange, the worst a meteor echo can do is double the amplitude for a few seconds. The shorter duration echoes will also be attenuated by the 0.48 -s coherent integration. As a check, hourly values of $\varepsilon^{\prime}$ near $100 \mathrm{~km}$ have been examined before, during, and after brief meteor showers (Quadrantids and Leonids) over 4-5 years (from 1998). In most cases, there is no discernable change in $\varepsilon^{\prime}$, but in the one or two cases where there is a hint of such variation, the $\varepsilon^{\prime}$ seems to be lower during the shower.

Restricting our attention to the height region $80-100 \mathrm{~km}$, therefore, we see from Figs. 1 and 2 that the expected semiannual variation is present in the lower thermosphere, with minima at the equinoxes. In winter and summer, zonal winds exist which affect the propagation of gravity waves from lower down up and into the lower thermosphere; while summer and winter states dictate an annual variation, the equinox states are characterised by low background winds due to the summer-winter (and vice-versa) wind reversals - a semiannual phenomenon. In the mid-mesosphere, the summer maxima are largely absent and the variation is annual. Figure 2 also illustrates this by showing simple a time series at 3 selected heights, and in which the absolute values are easier to discern.
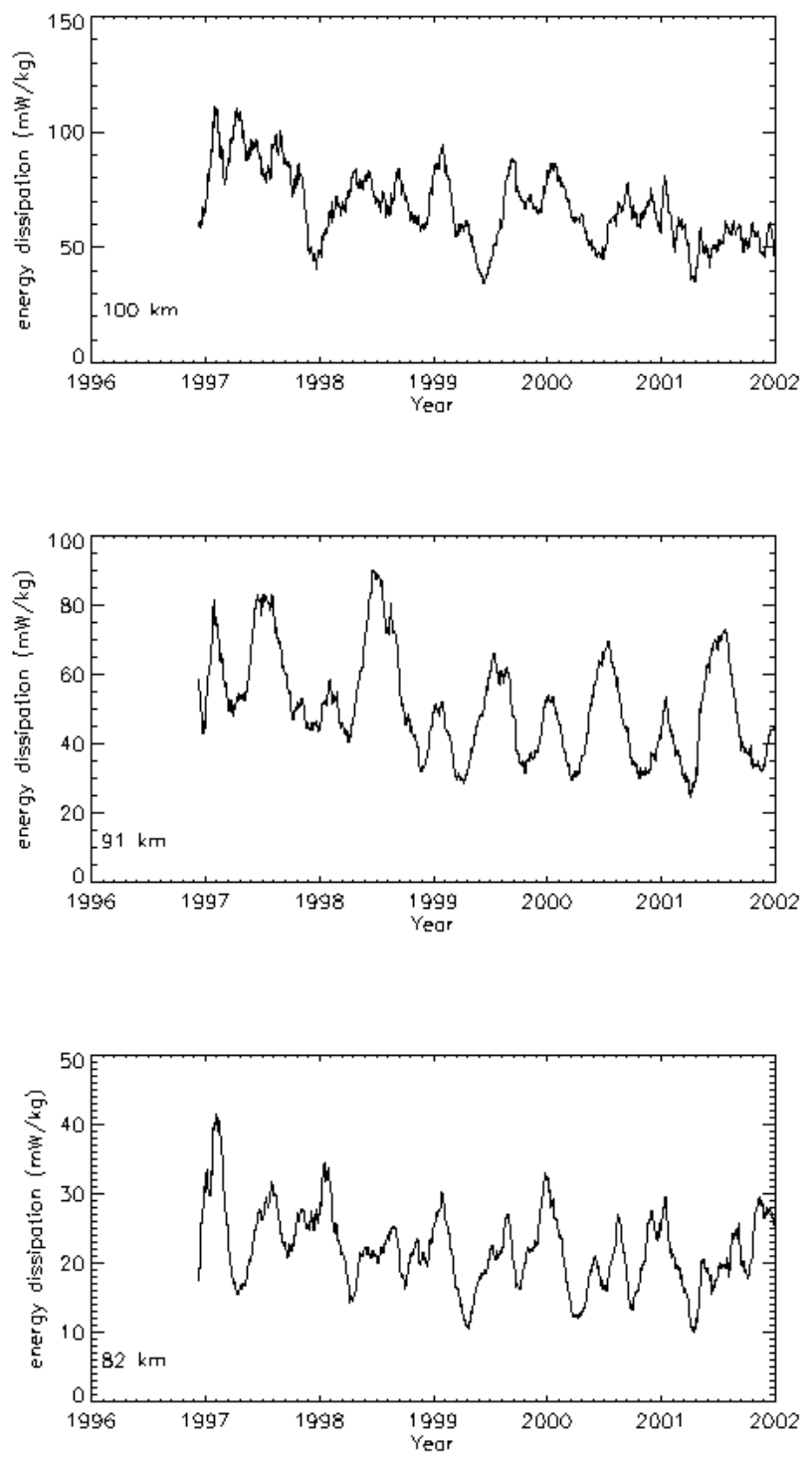

Fig. 2. Time series of $\varepsilon^{\prime}$ for the three selected heights 82,91 and $100 \mathrm{~km}$.

\section{Spectral analysis}

For each altitude, we have performed a spectral analysis of the data shown in Fig. 1. Since the time series are without substantial interruptions, we have chosen to interpolate the 1-day averages onto a 1-day grid. In this way we retain the original 1-day resolution but interpolate over any occasional missing days. Mean and trend removal and edge tapering using a Hanning window were performed, followed by Fourier transformation. Figures 3, 4 and 5 show the complete results divided into high, medium and low frequency regimes, to make the features more obvious. A similar study has been performed earlier by Hall et al. (1998), in which periodicities in both wind and $\varepsilon^{\prime}$, up to and including a 16-day period were investigated but using a shorter time series. Hall et al. (1998) examined the altitude and seasonal spectral charac- 


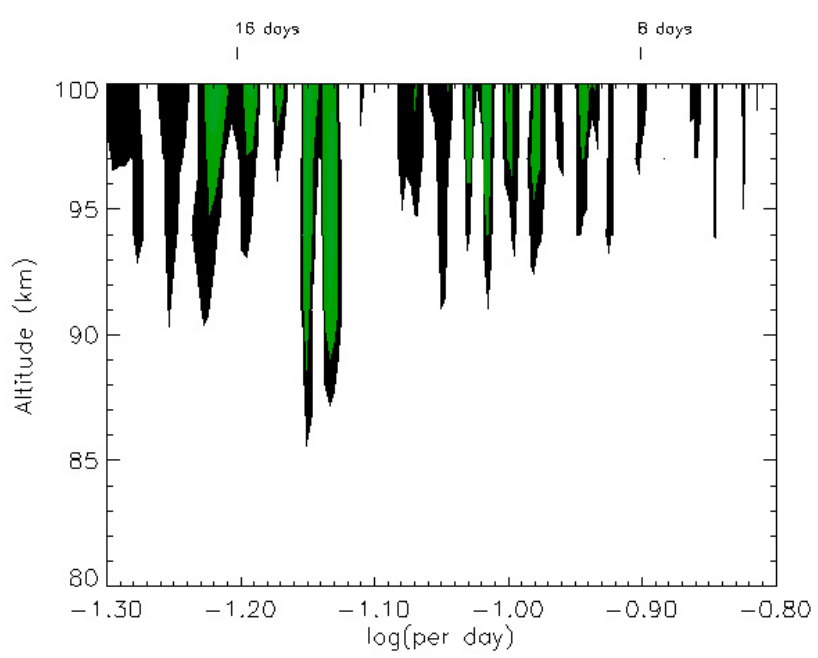

Fig. 3. One-dimensional power spectra from the data in Fig. 1. Absolute values are omitted to maintain clarity. Here the high frequency part of the spectra are shown. The abscissa indicates frequency in units of day ${ }^{-1}$, again, selected corresponding periods in days are indicated in the upper part of the panel. Contours show power spectral density in arbitrary units, the absolute values of which may be obtained from Fig. 6 .

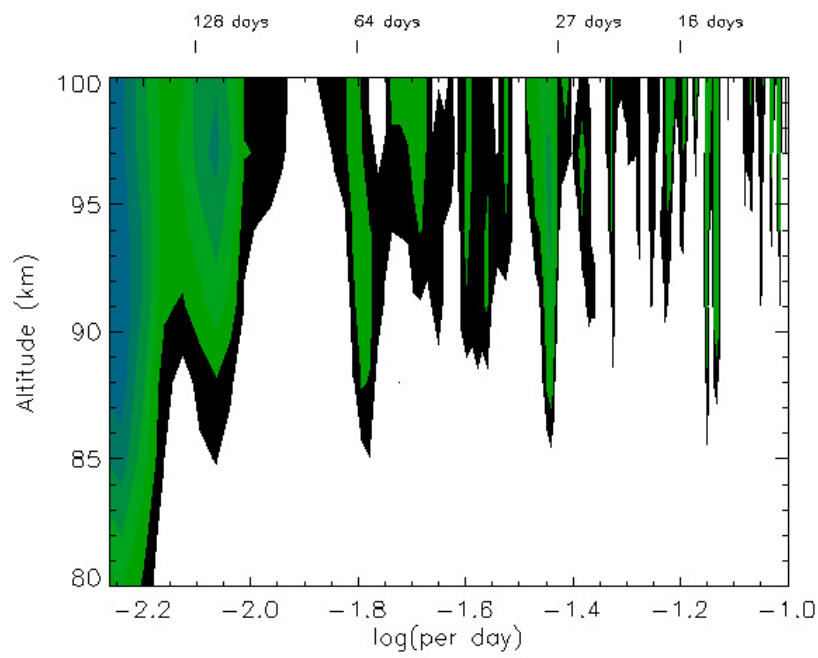

Fig. 4. As Fig. 3, but for a "middle" range of frequencies.

teristics of both MF radar determined winds and turbulence; periodicities ranged from those of tides, up to and including the 16-day Rossby modes. Using newer and longer time series, lower frequency features appear and it is these that we shall focus on in this paper. Visually, we can identify the following features:

1. Overall increase in amplitudes with height, with many periodicities not emerging below the mesopause region;

2. Evidence for the quasi-16-day wave above $85 \mathrm{~km}$, with a 13-15-day period and a maximum at 13.6 days;

3. Evidence for a $\sim 27$-day wave above $85 \mathrm{~km}$;

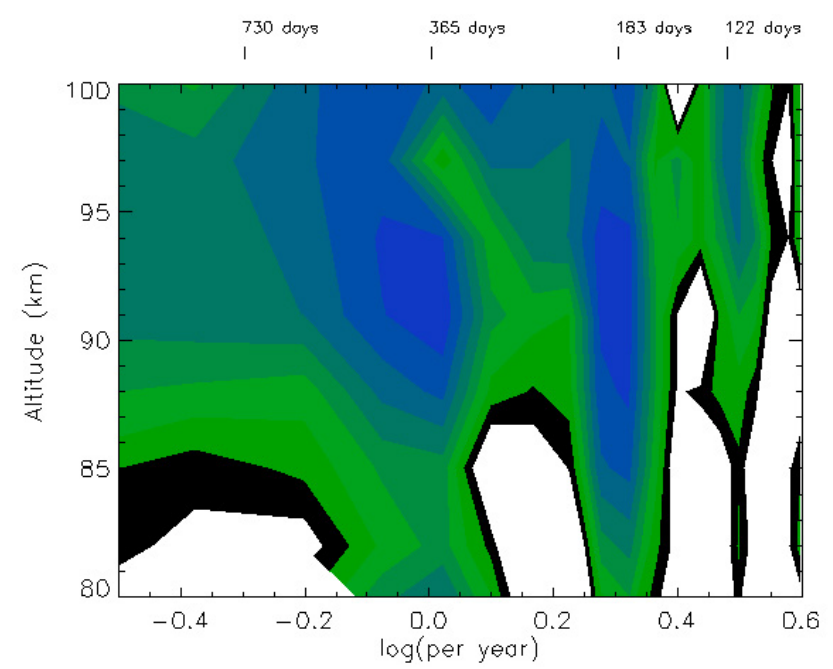

Fig. 5. As Fig. 3, but for low frequencies. Here, the abscissa has units of year ${ }^{-1}$.

4. Evidence for a 64-day wave, particularly prominent below $90 \mathrm{~km}$;

5. A terannual oscillation, primarily in the lower thermosphere, but also evident in the mesosphere;

6. SAO in the lower thermosphere (as anticipated);

7. Annual oscillation at all heights (as anticipated).

While some of the features in the figures could be anticipated, others could not. And the 14-day feature, which is most likely related to the 16-day planetary wave, is thought to be modulated by the QBO (Espy et al., 1997; Luo et al., 2000), which is a low-latitude phenomenon. For the 94-km virtual height, the results are shown in Fig. 6, the upper panel of which exhibits the full spectral resolution. While our primary tool for spectral analysis has been the Fourier transform, it is important to be able to assess the significance of the periodicities we identify. In order to accomplish this, we have performed Lomb-Scargle analyses of the time series in parallel and have obtained normalised power spectral density levels (occasionally referred to as "Lomb values") corresponding to different confidence levels (Hocke, 1998). We have scaled these confidence levels to the corresponding power spectral densities and indicated the $80 \%$ level on the one-dimensional spectrum (Fig. 6 and similarly for Fig. 9 described later). We see that the annual, semiannual, terannual ( $\sim 122$-day), $\sim 27$-day and $\sim 14$-day features are all above the $80 \%$ confidence level. It should be noted that the existence of the well-known annual and semiannual peaks sets the confidence level at a higher level than would be appropriate for shorter periods, so the true confidence level for periods shorter than the semiannual would be at a lower level than indicated in the upper panel of Fig. 6 .

To give further confidence in the $\sim 122$-day, $\sim 27$-day and $\sim 14$-day features, we have also employed the $a \chi^{2}$ approach (e.g. Beauchamp and Yuen, 1979). Having identified the 

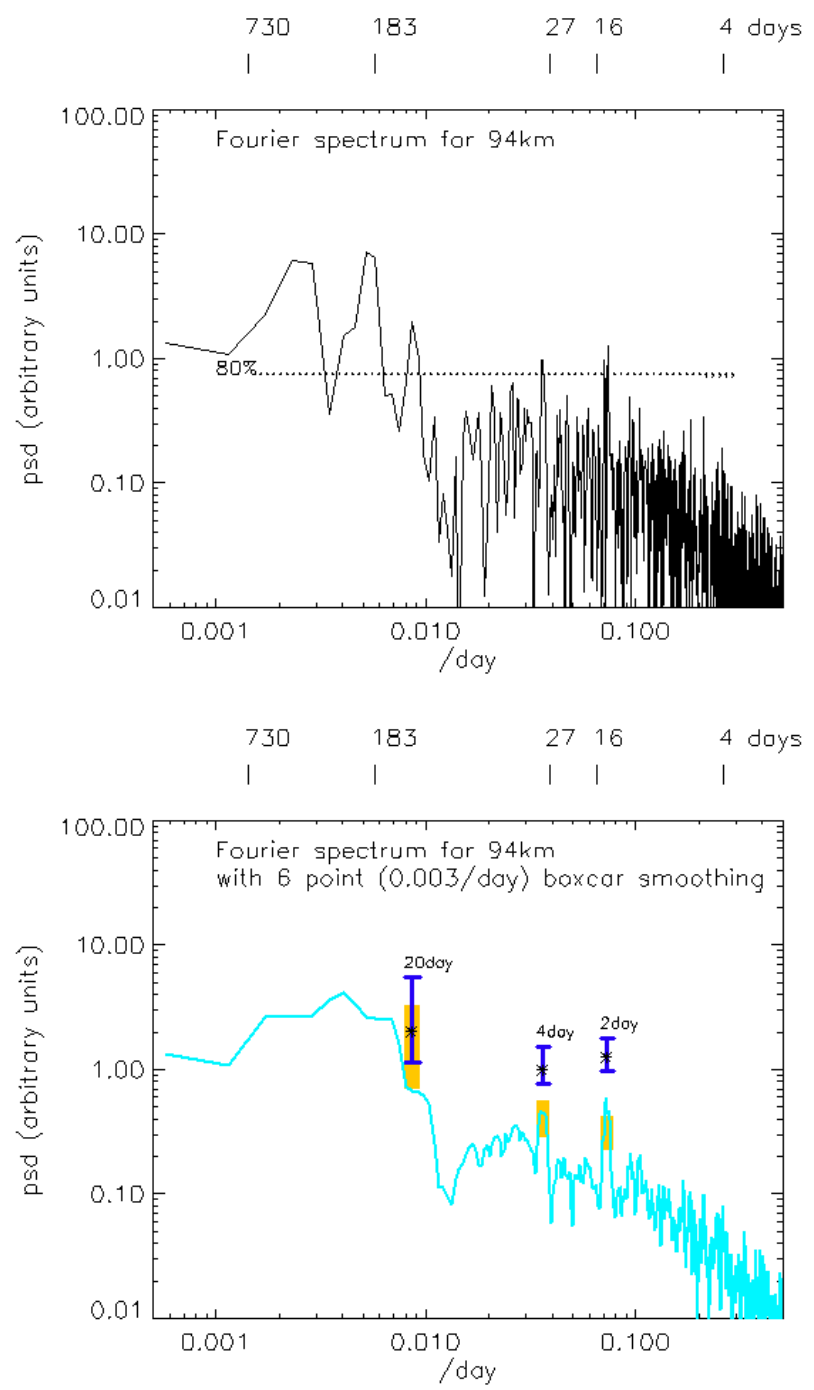

Fig. 6. Power spectrum of the time series at $94 \mathrm{~km}$. While frequency is given in day ${ }^{-1}$, the selected corresponding periods in days are indicated in the upper part of each panel. Upper panel: full resolution spectrum, the horizontal dotted line indicating the power spectral density corresponding to the Lomb $80 \%$ confidence level. Lower panel: Smoothed spectrum with $\chi^{2}$ accuracy limits for the $\sim 122$ day, $\sim 16$-day and $\sim 27$-day periods (see text for details).

peaks in the upper panel of Fig. 6, we selected the corresponding frequency bandwidths of \pm 10 days, \pm 2 days and \pm 1 day, respectively. From the corresponding numbers of degrees of freedom (and taking the Hanning window into consideration), we have obtained the accuracy limit factors for an $80 \%$ confidence level (e.g. Beauchamp and Yuen, 1979). We have applied these accuracy bands to both the peak values (for illustrative purposes) and (more correctly) to the mean of the spectrum within each frequency band. The lower panel of Fig. 6 shows the spectrum smoothed with a 6 point $(0.003$ day $^{-1}$ ) boxcar to help indicate the continuum. The original peaks for the $\sim 14-, \sim 27$ - and $\sim 122$-day periods are shown as asterisks. Accuracy limits are shown by I's (vertical error
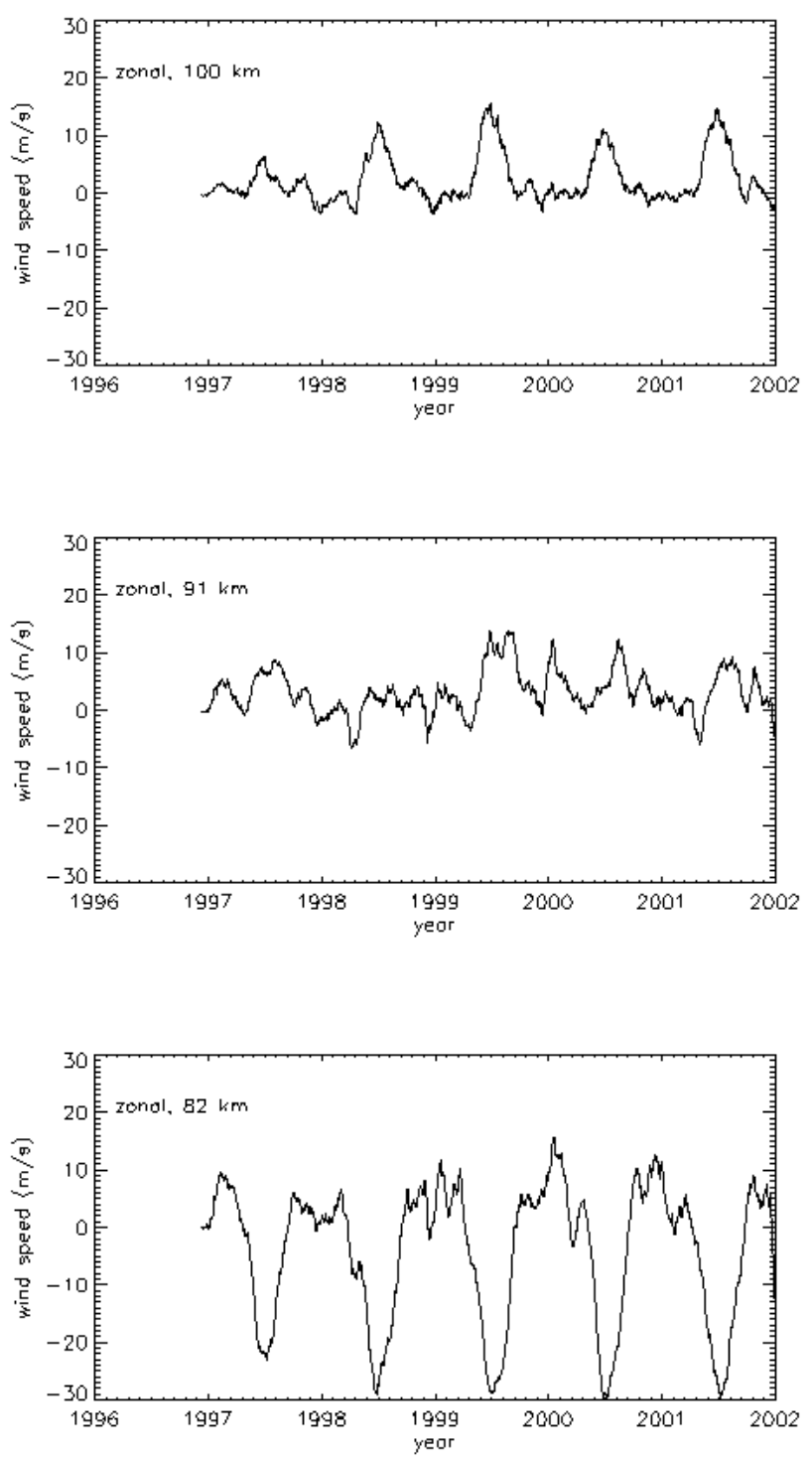

Fig. 7. As for Fig. 2, but for the zonal wind.

bars) for the peak values and by rectangles for the frequency band means. The widths of these symbols indicate the bandwidths, and their vertical extents show the accuracy bands. Both implementations of accuracy band occur above the continuum, giving us faith that the oscillations in question are, indeed, real.

\section{Interpretation}

To aid our interpretation of the energy dissipation periodicities, it will be necessary to examine the winds, in addition. Plots akin to those for $\varepsilon^{\prime}$ have, therefore, been constructed for each of the zonal and meridional components of the wind. Figures 7 and 8 show the horizontal wind in the time domain, while Figs. 9 and 10 show selected results of a spectral analysis of the zonal component performed in exactly the same manner as for $\varepsilon^{\prime}$. When considering winds 

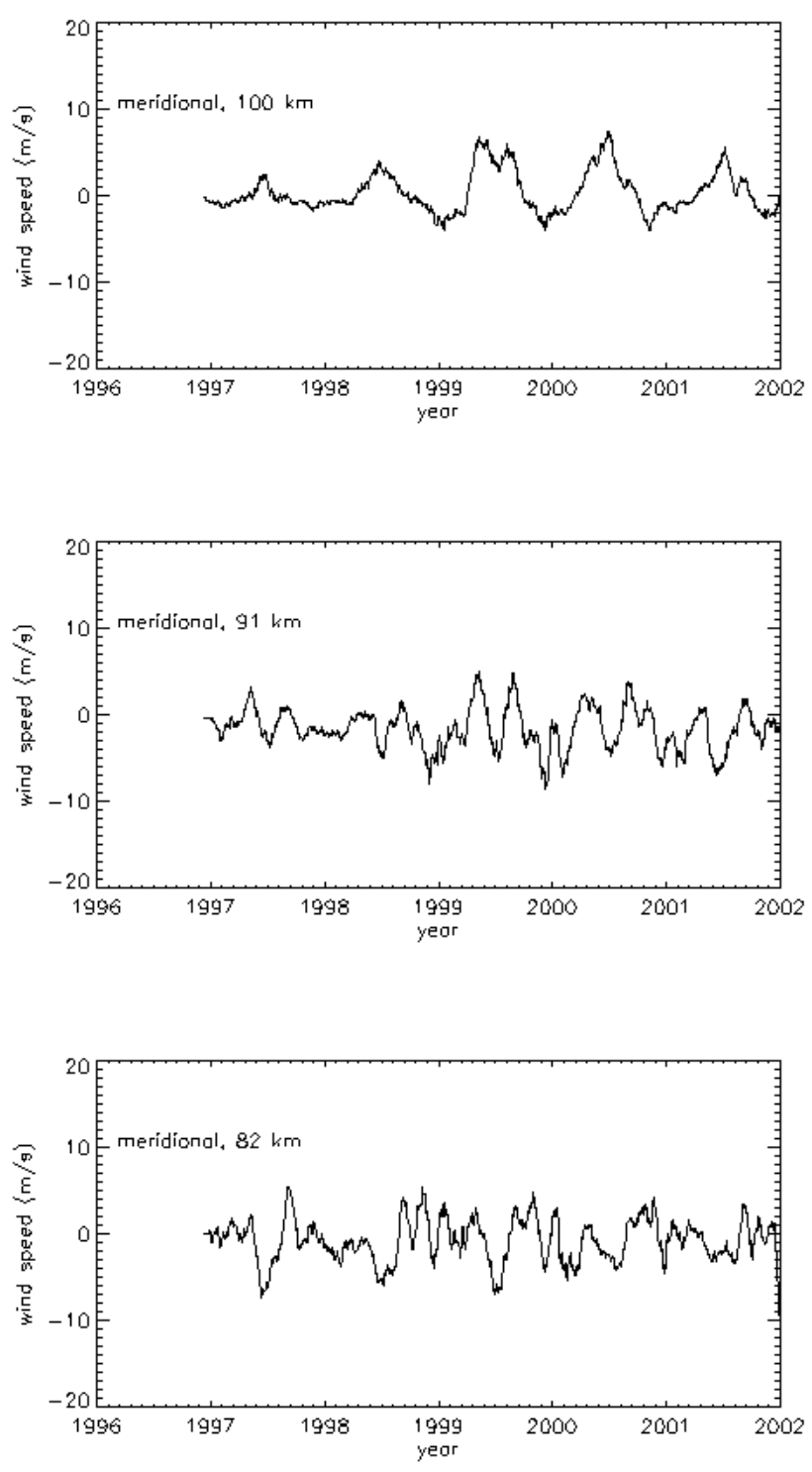

Fig. 8. As for Figs. 2 and 7, but for the meridional wind.

determined by the spaced-antenna technique used here, it should be pointed out that there has hitherto been a dispute as to their veracity. As stated earlier, partial reflection echoes above $90 \mathrm{~km}$ are frequently contaminated by meteor echoes, and also mechanisms other than neutral dynamics may be responsible for moving, dissipating and perturbing the radio wave scatterers at higher altitudes. Many comparisons have been made and are currently being made between MF radar derived winds and results from other instruments. Manson et al. (1992) compared winds from the instrument used in this study with other radar observations and with rocket soundings and with models (1999). Hussey et al. (2000) made comparisons with other radar determinations. Meek et al. (1997) show comparisons with satellite and optical methods. Manson et al. (2002) utilise MF radar derived dynamics to investigate gravity waves and subsequently to compare to modelling methods. There has been some discussion on the magnitudes of MF radar determined winds, particularly in the lower thermosphere (e.g. Manson et al., 1992). Strengths and weaknesses of the MF radar technique and the implicit assumptions are well discussed in these works and references therein; generally, however, determinations by MF radar are considered well representative of the neutral dynamics. Regardless, though, any systematic bias need not affect the periodicities in long-term data. We will proceed, therefore, by addressing each of the oscillations in turn.

\subsection{Annual and semiannual oscillations}

As stated earlier, these periodicities are anticipated and documented from before (Manson et al., 1981b; Namboothiri et al., 1994.). They are especially evident in the zonal wind (Fig. 7). These oscillations reach lower heights $(80 \mathrm{~km})$ in the wind field (Fig. 7, bottom) compared with $\varepsilon^{\prime}$ (Fig. 5), perhaps being related to the increase in gravity wave-induced turbulence with height. Typical seasonal variability special to $69^{\circ} \mathrm{N}$ have been documented by Lübken (1996) and Hall et al. (1999), encompassing both in situ and radar-based measurements of $\varepsilon$ and $\varepsilon^{\prime}$, respectively.

\subsection{Terannual and 64-day oscillations}

The emergence of a terannual oscillation is surprising, since a mechanism for driving the atmospheric dynamics at a $\sim 122$ day period is hard to envisage. At $85 \mathrm{~km}$, the summer mesopause, the feature in $\varepsilon^{\prime}$, is suppressed (Fig. 5), while above and to a lesser extent below, it is undeniable (there is a secondary maximum at $82 \mathrm{~km}$ ). Moreover, this oscillation is present in both zonal (Fig. 10) and meridional winds, although there is no indication of an $85-\mathrm{km}$ minimum. It is likely that dissipation processes are damped there due to gradients in temperature and density (at around $70^{\circ} \mathrm{N}$, the summer mesopause occurs near $85 \mathrm{~km}$ and above this height the temperature increases dramatically, with this increase in convective stability damping buoyancy motion). It is difficult to identify other observed (and explicable) periods that might interfere to produce this oscillation, and at this time we are unable to offer any physical explanation for the phenomenon. Similarly, we are unable to suggest any explanation for the 64-day feature; it is present in $\varepsilon^{\prime}$ at most altitudes and most evident in the wind field below $90 \mathrm{~km}$. It should be noted that if (for example) an annual oscillation is present which is not sinusoidal (for example, a boxcar form), ripples would appear in the spectra which would possibly include these periods. One would expect the amplitudes of these to be small, however, whereas the 122- and 64-day features we have identified here are quite strong. For the purposes of this study, we shall not pursue these periodicities further.

\subsection{Quasi-27-day oscillation}

An oscillation of around 27 days is a very clear feature of $\varepsilon^{\prime}$ (Fig. 4), being visible down to $85 \mathrm{~km}$, in contrast to most other periodicities. Similar maxima are clear in the individual $94 \mathrm{~km}$ wind spectra (Fig. 9), although not so prominent 


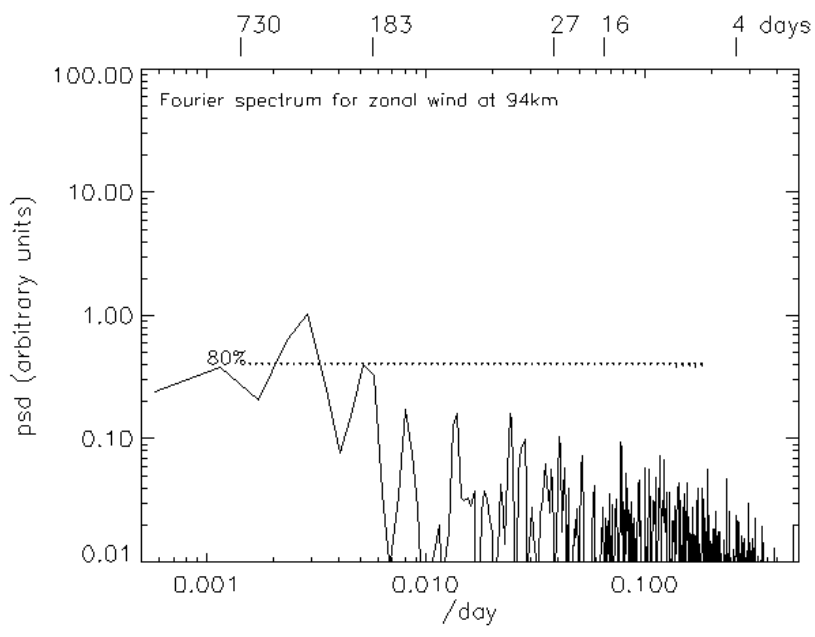

Fig. 9. As for Fig. 6 but for the zonal wind.

when examining the time-height portrayal (Fig. 10). That most amplitude is seen in the lower thermosphere leads us to suspect that this is a phenomenon driven by the auroral electrojet, for example around 100-120 km altitude (Kamide and Brekke, 1993), which, being dependent on the conductivity, is modulated by solar activity, thus having a period equal to the mean solar rotation. (27 days is the apparent solar rotation averaged over lower solar latitudes - the latitudes relevant to terrestrial physics; the solar rotation period at higher latitudes can be as high as 35 days.) That turbulence may be enhanced in the presence of ion drag has already been proposed by Hall (2001b). In this mechanism, strong electric fields (for example, $50 \mathrm{mVm}^{-1}$ - not uncommon in the auroral zone), drive the ions to a sufficient degree as to drag the neutral component into a state of dynamic instability. In this way, non-turbulent flow can be "pushed" into turbulence by the ion-drag, and also, existing neutral turbulence could be enhanced. However, due to the relatively short scale-height in the mesosphere/lower thermosphere, it is difficult for such effects to penetrate deep into the mesosphere. On the other hand, recent studies by Luo et al. (2001) have revealed this feature in the wind field at a variety of other geographic locations. Luo et al. (2001) propose that it could be related to the UV heating, although they point out that UV forcing (via direct heating of the stratospheric due to absorption of UV by ozone) does not directly affect the mesospheric wind field. It could be envisaged that increases in the stratospheric temperature would increase the mesospheric lapse rate and, through increased likelihood of convective instability, cause upwardly propagating gravity waves to dissipate before reaching the mesopause. This would, in turn, suggest that the energy dissipation rate in the upper mesosphere might be reduced, i.e. anticorrelated with the UV flux. To investigate this further, one would need to determine the temperature profile distortion due to the direct heating of the stratosphere and subsequently model the propagation of gravity waves from some lower boundary through the middle

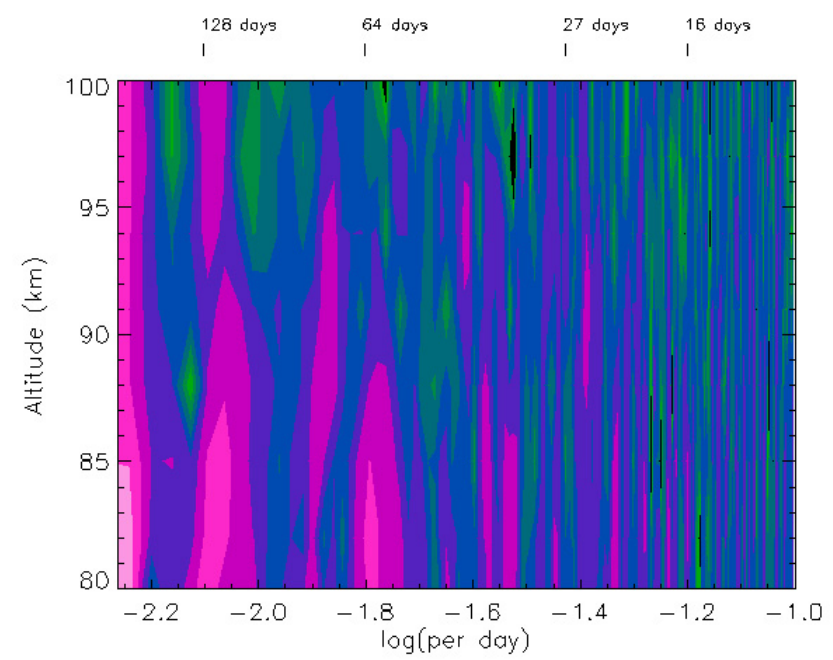

Fig. 10. As for Fig. 4 but for the zonal wind. Contours show power spectral density in arbitrary units, the absolute values of which may be obtained from Fig. 9.

atmosphere to the heights at which we see the 27-day feature. This task is beyond the scope of this paper, and furthermore, the high-latitude ozone (i.e. the constituent primarily responsible for UV-absorption), is notoriously highly variable. Also, Luo et al. (2001) noted that there was a weaker indication of correlations with the $K_{p}$ index in the lower thermosphere. Whereas Luo et al. (2001) employed cross-spectral analysis to search for correlations between solar parameters and winds, we have chosen to generate simpler scatter plots. For the test, we have selected the $100-\mathrm{km}$ virtual height $\varepsilon^{\prime}$ time series. Since the preceding figures indicated the 27-day period to be well-defined in frequency, we applied a digital band-pass filter to suppress periods shorter than 25 days and longer than 29 days. Figure 11 shows scatter plots of the result versus the Mg II UV index, the $10.7 \mathrm{~cm}$ flux, the mean $A p$ index and the summed $K_{p}$ index $\left(\sum K_{p}\right)$. Only the UV index and the 10.7 flux might conceivably be correlated with $\varepsilon^{\prime}$; however, due to the degree of scatter, we do not deign to obtain a regression line in either case. Although not shown here, we have repeated the test for a variety of altitudes and filter widths, including, of course, no filter at all, to no better success. The hint of anti-correlation of $\varepsilon^{\prime}$ with UV seen in Fig. 11 supports the hypothesis that stratospheric heating causes gravity wave energy to be deposited lower down, thus reducing the amount reaching the mesopause. However, we failed to note a positive correlation in the lowest altitudes of our observations. It would be possible to experiment with many different filtering philosophies, and, as stated above, we have experimented with varying the filter width to some degree. Both the filter coefficients (i.e. time domain) and the frequency response of the filter used here are shown in Fig. 12, and the reader is left to assess Fig. 11 in the light of the filter characteristics shown by Fig. 12.

Luo et al. (2001) reported a $0^{\circ}$ preferred phase between the 27-day wind oscillation and the $10.7 \mathrm{~cm}$ flux. This could 

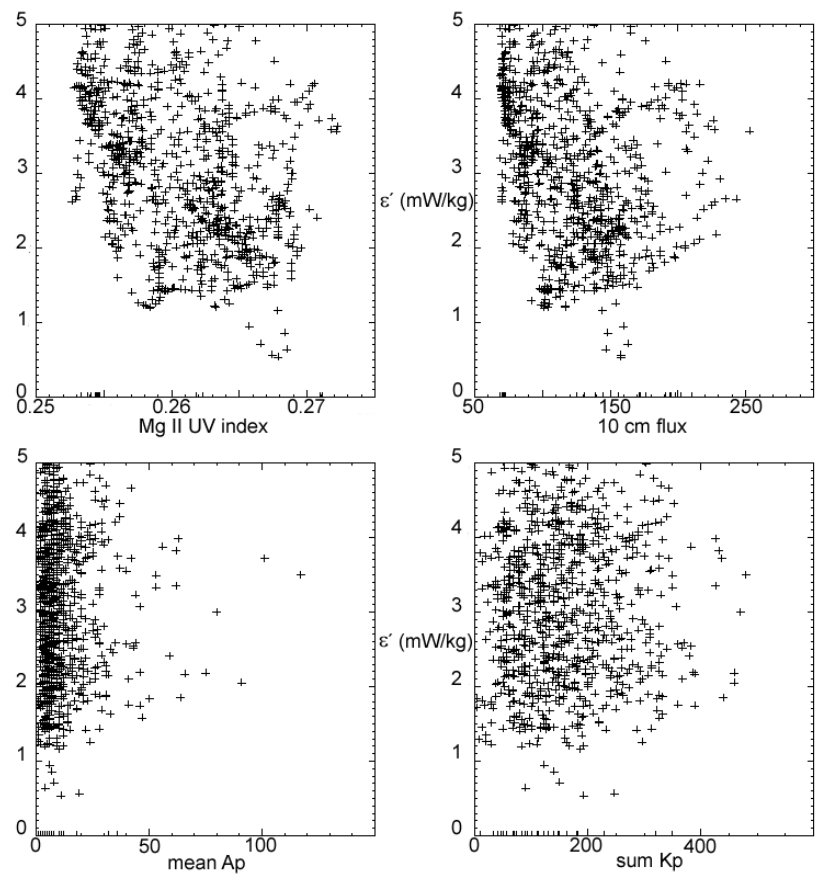

Fig. 11. Scatter plots of $\varepsilon^{\prime}$ versus various parameters which might characterize external forcing. Here, $\varepsilon^{\prime}$ from $100 \mathrm{~km}$ virtual height has first been filtered to suppress periods shorter than 25 days and longer than 29 days. Top left: $\varepsilon^{\prime}$ versus Mg II UV index; top right: versus $10.7 \mathrm{~cm}$ solar flux; bottom left: versus the mean $A p$; bottom right: versus $\sum K_{p}$.

be interpreted as confuting our "decreased convective stability" hypothesis, since the associated gravity wave dissipation would lead to a reduced momentum flux into the lower thermosphere and rather an absence of acceleration. However, it is the 27-day wind oscillation and not the background meanwind amplitude that correlates, and, therefore, the above argument is somewhat uncertain. Furthermore, the lack of correlation between $\varepsilon^{\prime}$ and the geomagnetic indices $A p$ and $\sum K_{p}$ do little to support the hypothesis of ion-drag destabilization of the neutral dynamics, which, as Hall and Aso (2000) suggest is likely to be a temporally and spatially limited phenomenon. Our results hitherto are, therefore, too inconclusive to give any strong support to any particular mechanism for the quasi-27-day oscillation. It is worth noting also that the nonlinear interaction between the 16- and 10-day normal mode planetary waves could also produce a 27 -day oscillation. Modelling of GCMs with and without 27-day solar inputs would be helpful in comparing these two mechanisms. Finally, one should not forget that the lunar tide exhibits a 29-day periodicity.

\subsection{Quasi 16-day oscillation}

This is most evident in the individual spectra, such as Fig. 6 and appears at periods slightly different from the nominal 16 days. This feature is well-known and is described in detail for mid-latitudes by Luo et al. (2000) and here we confirm its
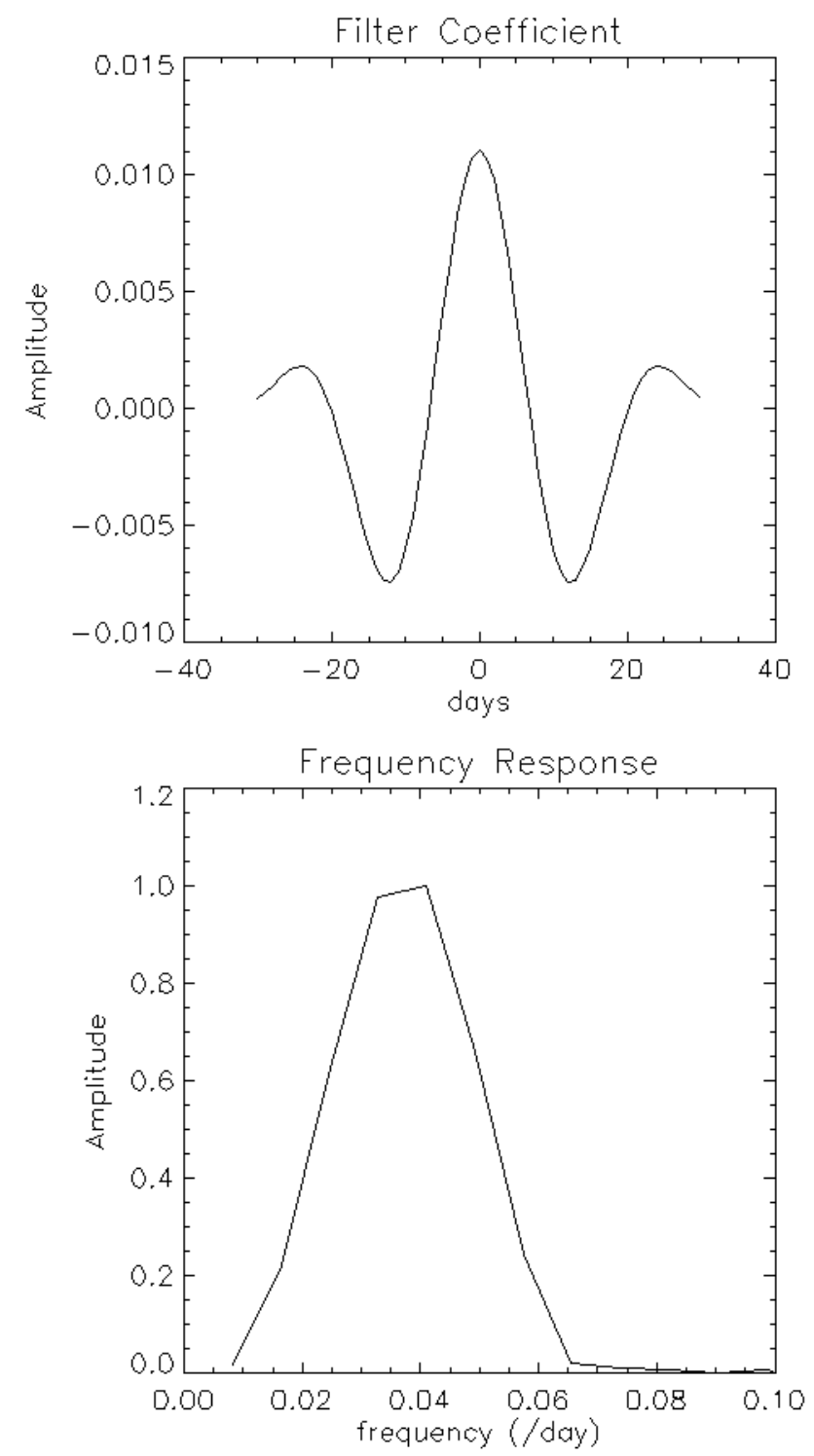

Fig. 12. Filter coefficients and frequency response for the 25-29 day band-pass filter used to isolate the quasi-27-day oscillation prior to its use for producing the scatter plots in Fig. 11.

presence at high latitude, in agreement with the more recent study by Luo et al. (2002). According to Meyer and Forbes (1997), the 16-day oscillation ought not to propagate above $100 \mathrm{~km}$; however, due to uncertainties in using MF radar data at such altitude, we have refrained from attempting to test this.

\subsection{General comments on the wind field}

Although it is the energy dissipation rates that are our main concern here, it is worth noting some features of the mean wind field. The apparent overall increase in the lower thermosphere jet in height is an interesting feature found, for example, by noting the altitude of the reversal in July (not visible in Fig. 7). An integration time of 5 min was used 
for the 1997, 1998 and 2001 data, whereas 2 min was used during 1999 and 2000. Also, a change in transmitter power at Troms $\varnothing$ late in 1998 could conceivably have affected the "height calibration" of the MF radar due to distortion of the transmitted pulse shape. The pulse shape is seldom a perfect Gaussian and is affected by the transmitter characteristics; the exact timing of the peak of the pulse in relation to its start could possibly change, thus affecting the measurement heights slightly. Even so, year-to-year differences are visible, suggesting this trend is a feature of the atmosphere. Transitions from westward to eastward jets in the lower thermosphere correspond to southward cells in the meridional component with corresponding changes in height. That the westward to eastward transitions correspond to a southward flow is a consequence of balance with gravity wave drag, explained by, for example, Manson et al. (1991), a study which included earlier data from Troms $\varnothing$ and stations at other latitudes. The trend, however, is not a feature of winds above Saskatoon at $52^{\circ} \mathrm{N}$, a station also included in Manson et al. (1991) and Luo et al. (2000, 2001 and 2002).

\section{Summary}

By performing spectral investigations of 5 years of the continually growing mesosphere/lower thermosphere energy dissipation estimate (parameterised by $\varepsilon^{\prime}$ ) data set from the MF-radar facility near Troms $\emptyset\left(69^{\circ} \mathrm{N}, 19^{\circ} \mathrm{E}\right)$, we have identified periodicities both anticipated and unexpected. The anticipated features included annual, semiannual and $\sim 16$ day oscillations, the unexpected features being terannual (i.e. 122-day) and 27-day oscillations. We are unable to explain the 27-day periodicity conclusively, but following the ideas of Luo et al. (2001) suggest that the cause lies in forcing from sub-mesosphere altitudes, or, alternatively, destabilisation of the neutral flow by ion-drag modulated by the solar-rotation. This latter hypothesis, inspired by Hall (2001b), is currently being further investigated in a parallel study comparing solar and geomagnetic parameters with $\varepsilon^{\prime}$ as an in-depth followup to Fig. 11 (Hall et al., 2002). The terannual oscillation remains a mystery.

Acknowledgements. Thanks go to the technical staff of Troms $\emptyset$ Geophysical Observatory for prolonged maintenance of the MFradar system described above, without which the time series used in this paper would be much impaired. Thanks also to T. A. Blix and U.-P. Hoppe for valuable advice.

Topical Editor D. Murtagh thanks D. Holdsworth and another referee for their help in evaluating this paper.

\section{References}

Beachamp, K. and Yuen, C.: Digital methods for signal analysis, 316 pp., George Allen and Unwin Ltd., London, UK, 1979.

Blamont, J.-E.: Turbulence in atmospheric motions between 90 and $130 \mathrm{~km}$ of altitude. Planet. Space Sci., 10, 89-101, 1963.

Blix, T. A., Thrane, E. V., and Andreassen, Ø.: In situ measurements of the fine-scale structure and turbulence in the mesosphere and lower thermosphere by means of electrostatic positive ion probes, J. Geophys. Res., 95, 5533-5548, 1990.

Briggs, B.H.: The analysis of spaced sensor records by correlation techniques, Handb. MAP, 13, 166-186, 1984.

Espy, P. J., Stegman, J., and Witt, G.: Inter-annual variations of the quasi 16-day oscillation in the polar mesospheric temperature, J. Geophys. Res., 102, 1983-1990, 1997.

Hall, C.M., Meek, C.E., and Manson, A.H.: Turbulent energy dissipation rates from the University of Tromsø/University of Saskatchewan MF radar, J. Atmos. Solar Terr. Phys., 60, 437 440, 1998.

Hall, C. M.: Virtual to true height correction for high latitude MF radar, Ann. Geophysicae, 16, 277-279, 1998.

Hall, C. M., Manson, A.H., and Meek, C.E.: Spectral Characteristics of spring arctic mesosphere dynamics, Ann. Geophysicae, 16, 1607-1618, 1998.

Hall, C. M., Hoppe, U.-P., Blix, T. A., Thrane, E. V., Manson, A. H., and Meek, C.E.: Seasonal variation of turbulent energy dissipation rates in the polar mesosphere: a comparison of methods, Earth Planet. Space, 51, 515-524, 1999.

Hall, C. M., Nozawa, S., Manson, A. H., and Meek, C. E.: Determination of turbulent energy dissipation rate from MF-radar determined velocity, Earth Planet. Space, 52, 137-141, 2000.

Hall, C. M. and Aso, T.: Identification of possible ion-drag induced neutral instability in the lower thermosphere over Svalbard, Earth Planet. Space, 52, 639-643, 2000.

Hall, C. M.: The Ramfjordmoen MF radar $\left(69^{\circ} \mathrm{N}, 19^{\circ} \mathrm{E}\right)$ : Application development 1990-2000, J. Atmos. Solar-Terr. Phys., 63, 171-179, 2001a.

Hall, C. M.: An analogy to the Reynolds number for the neutral gas component of a weak plasma, Ann. Geophysicae, 19, 131-134, $2001 b$.

Hall, C. M., Nozawa, S., Meek, C. E., and Manson, A. H.: On the response of fading times of upper homosphere radar echoes to solar and geomagnetic disturbances, Earth Planet. Space, 54, 699-705, 2002.

Hedin, A. E.: Extension of the MSIS thermosphere model into the middle and lower atmosphere, J. Geophys. Res., 96, 1159-1172, 1991.

Hocke, K.: Phase estimation with the Lomb-Scargle periodogram method, Ann. Geophysicae, 16, 356-358, 1998.

Hocking, W. K.: On the extraction of atmospheric turbulence parameters from radar backscatter Doppler spectra - I. Theory, J. Atmos. Terr. Phys. 45, 89-102, 1983.

Hocking, W. K.: The dynamical parameters of turbulence theory as they apply to middle atmosphere studies, Earth Planet. Space, 51, 525-541, 1999.

Holdsworth, D. A., Vincent, R. A., and Reid, I. M.: Mesospheric turbulent velocity estimation using the Buckland Park MF radar, Ann. Geophysicae, 19, 1007-1017 2001.

Hussey, G., Meek, C. E., Dieter, A., Manson, A. H., Sofko, G., and Hall, C.: A comparison of nothern hemisphere winds using SuperDARN meteor trail and MF radar wind measurements, J. Geophys. Res., 105, 18 053-18 066, 2000.

Kamide, Y. and Brekke, A.: Altitude variations of ionospheric currents at auroral latitudes, Geophys. Res. Lett., 20, 309-312, 1993.

Luo, Y., Manson, A.H., Meek, C.E., Meyer, C. K., and Forbes, J. M.: The quasi 16-day oscillation in the mesosphere and lower thermosphere at Saskatoon $\left(52^{\circ} \mathrm{N}, 107^{\circ} \mathrm{W}\right), 1980-1996$, J. Geophys. Res., 105, 2125-2138, 2000.

Luo, Y., Manson, A. H., Meek, C. E., Igarashi, K., and Jacobi, Ch.: 
Extra long period (20-40 day) oscillations in the mesospheric and lower thermospheric winds: observations in Canada, Europe and Japan, and considerations of possible solar influences, J. Atmos. Solar-terr. Phys., 63, 835-852, 2001.

Luo, Y., Manson, A.H., Meek, C.E., Meyer, C., Forbes, J., Burrage, M., Fritts, D. C., Hall, C. M., Hocking, W., MacDougall, J., Riggin, D., and Vincent, R.: The 16-day planetary waves:multiMF radar observations form the arctic to equator and comparisons with the HRDI measurements and GSWM modelling results, Ann. Geophysicae, 20, 691-709, 2002.

Lübken, F.-J.: Seasonal variation of turbulent energy dissipation rates at high latitudes as determined by in situ measurements of neutral density fluctuations, J. Geophys. Res., 102, 1344113 456, 1997.

Lübken, F.-J.: Rocket-borne measurements of small scale structures and turbulence in the upper atmosphere, Adv. Space Res., 17, (11), 25-35, 1996.

Manson, A.H., Meek, C.E., and Gregory, J.B.: Gravity waves of short period (5-90 min) in the lower thermosphere at $52^{\circ} \mathrm{N}$ (Saskatoon, Canada): 1978/1979. J. Atmos. Terr. Phys., 43, 3544, 1981a.

Manson, A.H., Meek, C. E., and Gregory, J. B.: Long-period oscillations in mesospheric and lower thermospheric winds (60$110 \mathrm{~km})$ at Saskatoon $\left(52^{\circ} \mathrm{N}, 107^{\circ} \mathrm{W}, L=4.3\right)$, J. Geomag. Geoelectr., 33, 613-621, 1981b.

Manson, A. H., Meek, C. E., Fleming, E., Chandra, S., Vincent, R. A., Phillips, A., Avery, S. K., Fraser, G. J., Smith, M. J., Fellous, J. L., and Massebeuf, M.: Comparison between satellite-derived gradient winds and radar-derived winds from the CIRA-86, J. Atmos., Sci., 48, 411-428, 1991.

Manson, A. H., Meek, C. E., Brekke, A., and Moen, J.: Mesosphere and lower thermosphere $(80-120 \mathrm{~km})$ winds and tides from near Troms $\varnothing\left(70^{\circ} \mathrm{N}, 19^{\circ} \mathrm{E}\right)$ : comparisons between radars (MF, EISCAT, VHF) and rockets, J. Atmos. Terr. Phys., 54, 927-950, 1992.

Manson, A.H., Meek, C.E., Hagan, M., Hall, C.M., Hocking, W. K., MacDougall, J., Franke, S., Fritts, D. C., Riggin, D., and
Vincent, R.: Seasonal variations of the semi-diurnal and diurnal tides in the MLT: Multi-year MF radar observations from 2$70^{\circ} \mathrm{N}$ and the GSWM, J. Atmos. Solar-Terr. Phys., 61, 809-828, 1999.

Manson, A.H. Meek,, C.E., Koshyk, J., Franke, S., Fritts, D. C., Riggin, D., Hall, C.M., Hocking, W.K., MacDougall, J., Igarashi, K., and Vincent, R. A.: Gravity wave activity and dynamical effects in the middle atmosphere $(60-90 \mathrm{~km})$ : observations from an MF/MLT radar network, and results from the Canadian Middle Atmosphere Model (CMAM), J. Atmos. Solar-Terr. Phys., 64, 65-90, 2002.

Meek, C.E.: An efficient method for analysing ionospheric drifts data, J. Atmos. Terr. Phys., 42, 835-839, 1980.

Meek, C. E.: Triangle size effect in spaced antenna wind measurements, Radio Sci., 25, 641-648, 1990.

Meek, C.E. and Manson, A. H.: MF radar spaced antenna experiment: wind variance vs. record length, J. Atmos. Solar-Terr. Phys., 63, 181-191, 2001.

Meek, C.E., Manson, A.H., Burrage, M.D., Garbe, G., and Cogger, L. L.: Comparisond between Canadian prairie MF radars, FPI (green and OH lines) and UARS HRDI systems, Ann. Geophysicae, 15, 1099-1110, 1997.

Meyer, C.K. and Forbes, J.M.: Natural oscillations of the ionosphere-thermosphere-mesosphere (ITM) system, J. Atmos. Solar-Terr. Phys., 59, 2185-2202, 1997.

Namboothiri, S.P., Manson, A. H., and Meek, C. E.: E-region real heights and their implications for MF radar-derived wind and tidal climatologies, Radio Sci., 28, 187-202, 1993.

Namboothiri, S. P., Meek, C. E., and Manson, A. H.: Variations of mean winds and solar tides in the mesosphere and lower thermosphere over timescales ranging from 6 months to $11 \mathrm{yr}$ : Saskatoon, $52^{\circ} \mathrm{N}, 107^{\circ} \mathrm{W}$, J. Atmos. Terr. Phys., 56, 1313-1325, 1994.

Roper, R. G.: On the radar estimation of turbulence parameters in a stably stratified atmosphere, Radio Sci., 35, 999-1008, 2000.

Schlegel, K., Thrane, E. V., and Brekke, A.: Partial Reflection in the auroral D-region explained in terms of acoustic waves, J. Atmos. Terr. Phys., 42, 809-814, 1980. 